\title{
Mechanism of Baoyuan Decoction in the Treatment of Chronic Heart Failure Based on Network Pharmacology
}

\author{
XIANYING YUAN, LINA LV AND R. WANG ${ }^{*}$
}

College of Traditional Chinese Medicine, ${ }^{1}$ Department of First Clinical Nursing, School of Nursing, Changchun University of Chinese Medicine, Jilin, Changchun 130117, PR China

\section{Yuan et al.: Baoyuan Decoction in the Treatment of Chronic Heart Failure}

This study aims to explore the material basis and mechanism of Baoyuan decoction in the treatment of chronic heart failure by network pharmacology and molecular docking technology. There are 144 effective active ingredients and 253 targets in Baoyuan decoction. The key ingredients mainly include flavonoids such as quercetin, naringenin and glazin. There are 240 intersecting targets between Baoyuantang and chronic heart failure, and its core targets include jun proto-oncogene, signal transducer and activator of transcription 3, tumor protein 53, transcription factor p65, mitogen-activated protein kinase 14, estrogen receptor 1, etc. The gene ontology and kyoto encyclopedia of genes and genomes enrichment analysis results show that it mainly involves 2475 biological processes, 104 cell components, 253 molecular functions and 174 related signals pathway. It mainly regulates the evolutionary processes including nutritional response, inflammatory response, oxidative stress response, apoptosis signal pathway etc., through the cell components such as cytoplasm, cell membrane and synapse, and exerts the molecular functions of DNA binding transcription factor, RNA polymerase II specific DNA binding transcription factor, serine/ threonine kinase activity, ubiquitin-like protein ligase binding, through phosphatidylinositol 3 kinaseprotein kinase B, Mitogen-activated protein kinase 14, Advanced glycation end products-receptor for AGE, Interleukin-17, apoptosis, cancer and other signaling pathways to play a role in the treatment of chronic heart failure effect.

Key words: Baoyuan decoction, chronic heart failure, oxidative stress, inflammatory reaction, cancer pathway

Chronic Heart Failure (CHF) is a clinical syndrome caused by abnormalities in the structure and function of the heart that cause systolic or diastolic dysfunction, which is mainly characterized by dyspnea, edema and restricted activity ${ }^{[1]}$. CHF is often accompanied by multiple organ failure, secondary hyperthyroidism, anemia, cancer and other diseases, which cause the increase of pro-inflammatory cytokines, electrolyte disturbances, calcium and phosphorus imbalances, neurohormonal disorders, which further aggravate the condition and poor prognosis ${ }^{[2]}$. The number of $\mathrm{CHF}$ patients in China is more than 4 million, which is an important factor of death of cardiovascular patients. CHF is still a major public health problem in China ${ }^{[3,4]}$.

CHF belongs to the categories of "palpitations" and "edema" in Traditional Chinese Medicine (TCM). TCM believes that CHF is a syndrome of deficiency and superficiality. The deficiency of its origin is mainly qi deficiency and it always runs through the entire course of CHF. Baoyuan decoction is a classic prescription for the treatment of deficiency and decay of vital energy. It is derived from the "Bo Ai Xin Jian" by Wei Zhi in the Ming dynasty. It is composed of four herbs: Astragalus, ginseng, licorice and cinnamon. It has the effect of invigorating temperature and yang and is the first of many Qi invigorating prescriptions. It is widely used in the clinical treatment of cardiovascular diseases and the treatment of CHF has a significant effect.

At present, there are few basic researches on the treatment of CHF with Baoyuan decoction. Therefore, this study uses network pharmacology methods to systematically conduct data mining on the correlation between Baoyuan decoction and CHF and explore the material basis and effect of Baoyuan decoction in the

*Address for correspondence

E-mail: wangruonan4027@163.com 
treatment of CHF. The mechanism provides the basis for the clinical medication and experimental research of Baoyuan decoction in cure of CHF.

\section{MATERIALS AND METHODS}

\section{Active ingredients and targets of Baoyuan decoction:}

Adopt Traditional Chinese Medicine System Pharmacology Database (TCMSP, https://www. tcmspw.com/tcmsp.php) to retrieve all active pharmaceutical ingredients and targets in Baoyuan decoction (astragalus, ginseng, cinnamon, licorice). In the pharmacokinetic characteristics of TCM, the oral bioavailability $(\mathrm{OB}) \geq 30 \%$ and the Drug-Like activity (DL) $\geq 0.18$ are used as the screening threshold. At the same time, according to the different chemical components and biological effects of the TCM, the final analysis of the TCM activity is a determined element. Import the acquired targets data into UniProt (https:// www.uniprot.org/), limit its gene source to humanderived genes that have been verified and finally obtain the target genes of the active ingredients of Baoyuan decoction.

\section{Screening of CHF-related targets:}

Use GeneCards database (https://www.genecards.org/), Online Mendelian Inheritance in Man (OMIM) database (https://www.omim.org/), Therapeutic Targets Database (TTD) (http://db.idrblab.net/ttd/), Pharmacogenomics Knowledge Base (PharmGKB) database (https:// www.pharmgkb.org/), DrugBank database (https:// go.drugbank.com/), then search keyword "Chronic heart failure", obtain CHF related genes and display the number of targets in the collection in the form of a Venn diagram.

\section{Baoyuan decoction and CHF target network construction:}

Adopt the obtained Baoyuan decoction and CHF gene targets to show the number of intersection targets of Baoyuan decoction-CHF disease in the form of a Venn diagram. Use Cytoscape 3.8.0 to display the network interaction diagram between the effective components of Baoyuantang and CHF disease targets and classify them according to the degree value.

\section{Construction of Protein-Protein Interaction (PPI) network:}

Import the intersection target of Baoyuantang and $\mathrm{CHF}$ into the Search Tool for the Retrieval of Interacting Genes/Proteins (STRING) database (https://www. string-db.org/), limit the species as "Homo sapiens", set the minimum interaction value to 0.950 and build the final protein interaction network picture. Import PPI results into Cytoscape 3.8.0 software, select Betweenness (BC), Closeness (CC), Degree (DC), Eigenvector (EC), Local Average Connectivity based method (LAC), Network (NC) and other parameters to obtain PPI network core target.

\section{Gene Ontology (GO) and Kyoto Encyclopedia of Genes and Genomes (KEGG) enrichment analysis:}

Use $\mathrm{R} \times 64$ 4.0.2 software for GO and KEGG enrichment analysis of the target symbol and Identity Document(ID) that have been screened and set the screening condition as $p<0.05$. GO enrichment analysis includes three parts which are as follows: Biological Process (BP), Cellular Component (CC) and Molecular Function (MF). Obtain the key target of Baoyuan decoction for CHF treatment. KEGG enrichment analysis obtains the most relevant signal pathways, draws bubble charts and histograms, the larger the node, the more enriched targets and the possible mechanism of Baoyuan decoction in treating CHF is analyzed.

\section{Molecular docking verification:}

The 6 key components screened out by the PPI network are molecularly docked with the core target. Define "Human" in the UniProt database, search the core target protein ID, search the Protein Data Bank (PDB) database and PubChem database to obtain its two Dimensional (2D) structure, use ChemBio3D Ultra 14.0 to convert its 2D structure to three Dimensional (3D) structure and import the protein receptor and ligand structure docking with PyMOL software to obtain the affinity value.

\section{RESULTS AND DISCUSSION}

Active ingredients of Baoyuan decoction is shown below. Through TCMSP database search, a total of 657 compounds of Baoyuan decoction were obtained, including 87 astragalus, 190 ginseng, 100 cinnamon and 280 licorice. After screening, 144 active ingredients were finally obtained, including 20 astragalus and 22 ginseng, 10 cinnamon and 92 licorice. A total of 5031 targets of Baoyuan decoction were obtained using the TCMSP database, including 953 astragalus, 748 ginseng, 824 cinnamon and 2506 licorice. By removing duplicate targets, 253 were finally obtained, as shown in Table 1. Use Cytoscape 3.8.0 to construct Baoyuan decoction-active ingredient map of the drug, as shown in fig. 1. 
TABLE 1: BAOYUAN DECOCTION COMPOUNDS, ACTIVE INGREDIENTS AND TARGET INFORMATION

\begin{tabular}{lccc}
\hline TCM & Compound (a) & Active ingredients (a) & Target point (a) \\
\hline Astragalus & 87 & 20 & 953 \\
Ginseng & 190 & 22 & 748 \\
Cinnamon & 100 & 10 & 824 \\
Licorice & 280 & 92 & 2506 \\
\hline
\end{tabular}

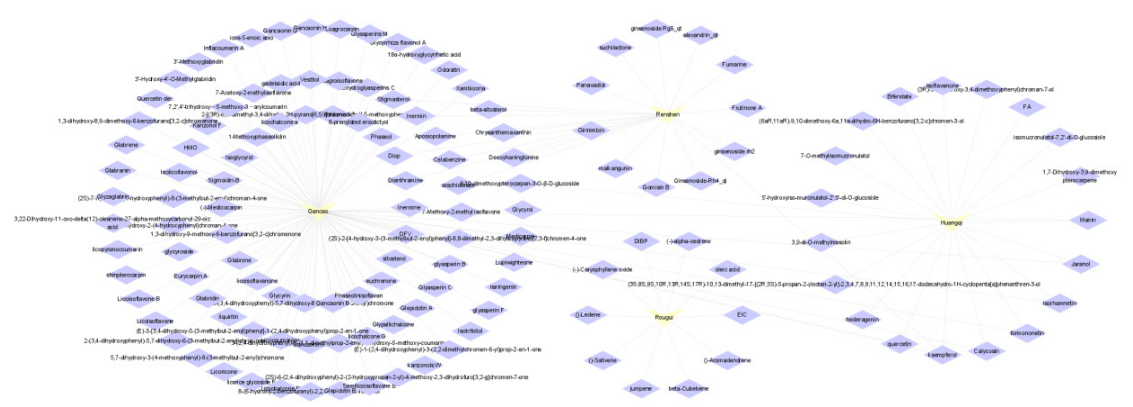

Fig. 1: Baoyuan decoction-network diagram of active ingredients of medicine

The intersection of Baoyuantang's target and CHF gene target is explained. Search for the keyword "Chronic heart failure" through GeneCards, OMIM, TTD, PharmGKB, DrugBank gene database and obtain a total of 9424 CHF related targets, as shown in fig. 2. After matching 253 Baoyuantang drug targets with 9424 CHF related gene targets using Venn software, a total of 240 intersection targets were obtained, which is shown in fig. 3.

Baoyuantang-CHF target network construction is explained briefly. Use Cytoscape 3.8.0 to combine the active substance of Baoyuan decoction with the target of CHF to construct a visualization map of "drug activity-target". There are a total of 363 nodes and 1773 edges in the graph of which the $\mathrm{V}$ shape represents the active ingredient of the drug, a total of 123; the diamond represents the gene target, a total of 240 and node size represents degree size. Through analysis, it is found that Baoyuan decoction active ingredients are mainly quercetin, kaempferol, formononetin, naringenin, isorhamnetin and Pueraria lobata, glabridin, oleic acid, 7-methoxy-2-methyl isoflavone, 7-O-methyl isoflavone (7 -O-methylisomucronulatol), etc., which mainly act on Ethylene Response Sensor 1 (ERS1), Nitric Oxide Synthase 2 (NOS2), Mitogen-activated protein kinase 14 (MAPK14), Jun Proto-Oncogene, AP-1 Transcription Factor Subunit (JUN) and other targets, as shown in fig. 4.

PPI network construction was explained below. The 240 intersection targets obtained through the screening of $\mathrm{R} \times 64$ 4.0.2 software are uploaded to the STRING database to format PPI network. The circle is the target and the edge is the protein interaction relationship, including 239 nodes, 4134 edges and the average number of nodes is 34.6 , the average number of local clusters is 0.564 and the PPI enrichment $p$ value: $<1.0 \mathrm{e}-16$, as shown in fig. 5. Using Cytoscape 3.8.0, 12 network core targets based on the degree value are obtained which are Signal Transducer and Activator of Transcription 3 (STAT3), Fos Proto-Oncogene (FOS), Tumor protein 53 (TP53), c-Jun amino Terminal kinase (JNK), Mitogen-Activated Protein Kinase 1 (MAPK1), Mitogen-Activated Protein Kinase 3 (MAPK3), Transcription factor p65 (RELA), Estrogen Receptor 1 (ESR1), RB Transcriptional Corepressor 1 (RB1), Cyclin Dependent Kinase Inhibitor 1A (CDKN1A), MAPK14 and Cyclin D1 (CCND1) (fig. 6).

240 targets were analyzed by GO enrichment and a total of 2833 results were obtained. There are 2475 BPs of Baoyuan decoction for treating CHF, which mainly include nutritional level, metal ion response, oxidative stress response, lipopolysaccharide response, steroid hormone response, response to bacteria-derived molecules and regulation of small molecule metabolism. Response to Reactive Oxygen Species (ROS) can regulate apoptosis pathways, lipid metabolism, inflammation and DNA binding transcription factor activity, etc. There are 104 CC, mainly includes membrane raft, membrane area, cytoplasm, nucleus, synapse, cell-basal junction, outer mitochondrial membrane, endoplasmic reticulum lumen, etc., and a total of 253 MFs which mainly includes DNA binding transcription factors, amides, RNA polymerase II specific DNA binding transcription factors, peptides, ubiquitin-like protein ligase, growth factors, etc., serine/threonine kinase activity, receptor ligand activity, etc., (fig. 7). 
www.ijpsonline.com

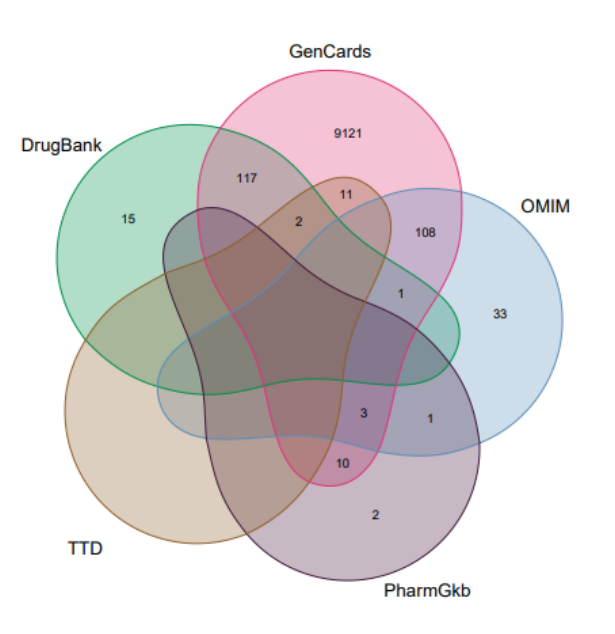

Fig. 2: Venn map of CHF disease gene

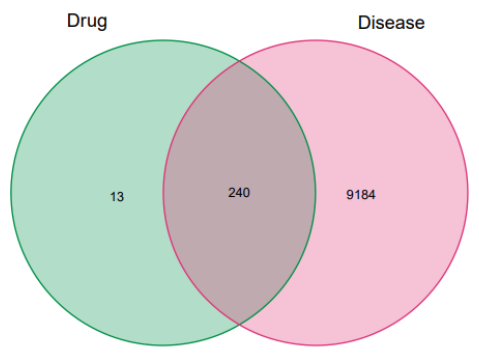

Fig. 3: Baoyuan decoction-venn map of CHF disease gene

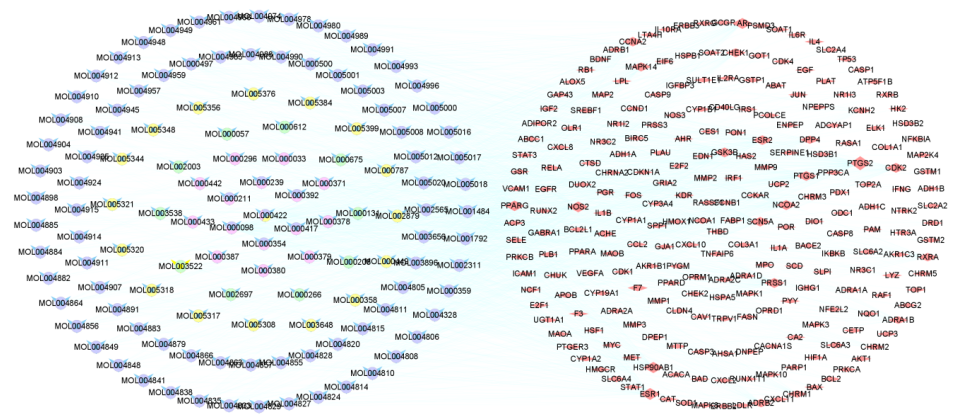

Fig. 4: Baoyuantang-CHF intersection target network diagram

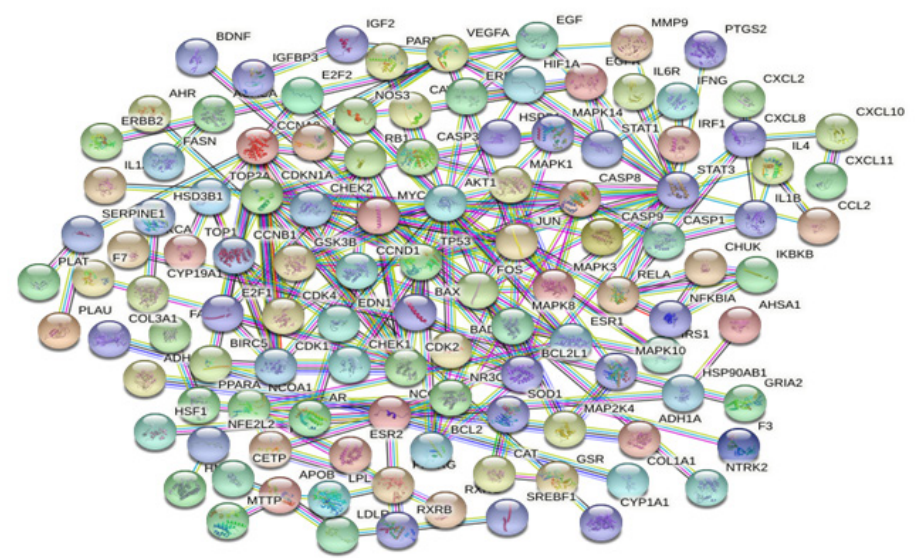

Fig. 5: PPI network diagram 

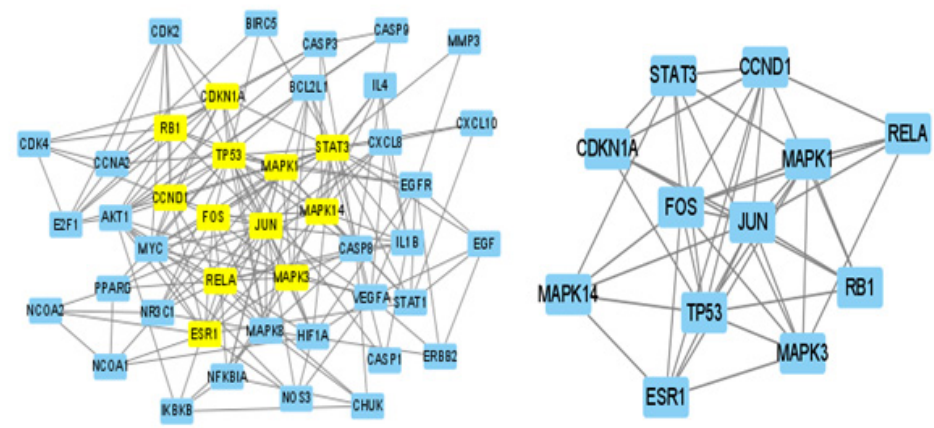

Fig. 6: Screening of core targets
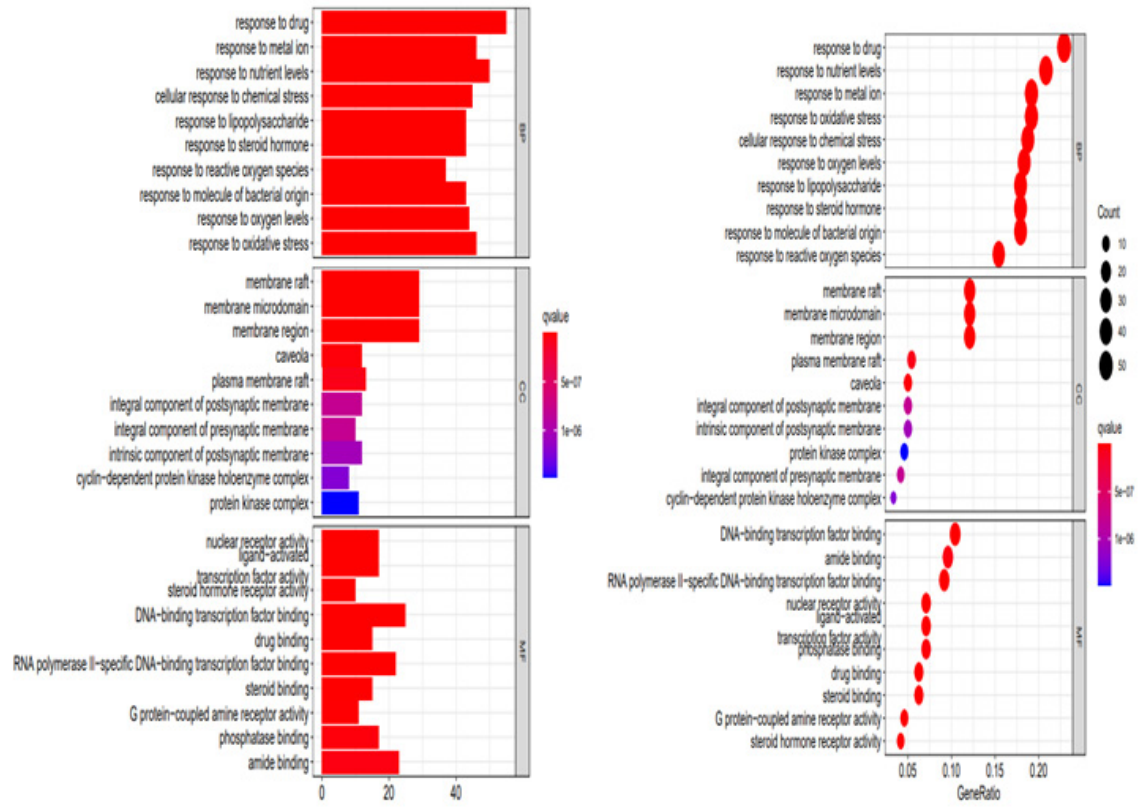

Fig. 7: GO enrichment analysis of the core target of Baoyuan decoction in the treatment of CHF

Through the KEGG collateral analysis of the target protein, 174 related signal pathways of Baoyuan decoction for treating CHF were obtained and the first 30 pathways were selected, as shown in fig. 8 which mainly involved Phosphatidylinositol 3 Kinase-protein kinase B (PI3K-Akt), MAPK, Advanced Glycation End Products-Receptor for AGE (AGE-RAGE), Tumour Necrosis Factor (TNF), Interleukin-17 (IL-17), apoptosis and other signaling pathways; liver cancer, gastric cancer, colorectal cancer, pancreatic cancer and other cancer pathways; hepatitis B, various infections, etc., but also with Renin-Angiotensin System (RAS), Cyclic Adenosine Monophosphate (cAMP), Toll-like receptors, p53, Janus Kinase and Signal Transducer and Activator of Transcription (JAK-STAT), Nuclear factor kappa-light-chain-enhancer of activated B cells $(\mathrm{NF}-\kappa \beta)$, T cell receptors, etc. The pathways are closely related, suggesting that Baoyuan decoction may exert a therapeutic effect on CHF through the above related pathways.
Using PyMOL software, the first 6 key active ingredients (quercetin, kaempferol, formononetin, isorhamnetin and glazin) screened out by Baoyuan decoction was molecularly docked with the core targets in the PPI network obtain the affinity value (Table 2 ). When the result of affinity $<-5$, it means that the molecule has better binding activity to the target and the smaller the affinity value, the stronger the binding activity of the active ingredient and the receptor ${ }^{[5,6]}$. According to the docking results, it was found that the binding activity of quercetin and MAPK1 and naringenin and MAPK1 was the best and the binding energy was $-9.0 \mathrm{kcal} / \mathrm{mol}$. In addition, quercetin and RB1, JUN; kaempferol and JUN; naringenin and MAPK3, ESR1; formononetin and MAPK14, ESR1; isorhamnetin and ESR1, glazin and MAPK14 all showed a good combination. The activity and binding energy are all $<-8.0 \mathrm{kcal} / \mathrm{mol}$ or less, as shown in fig. 9. 

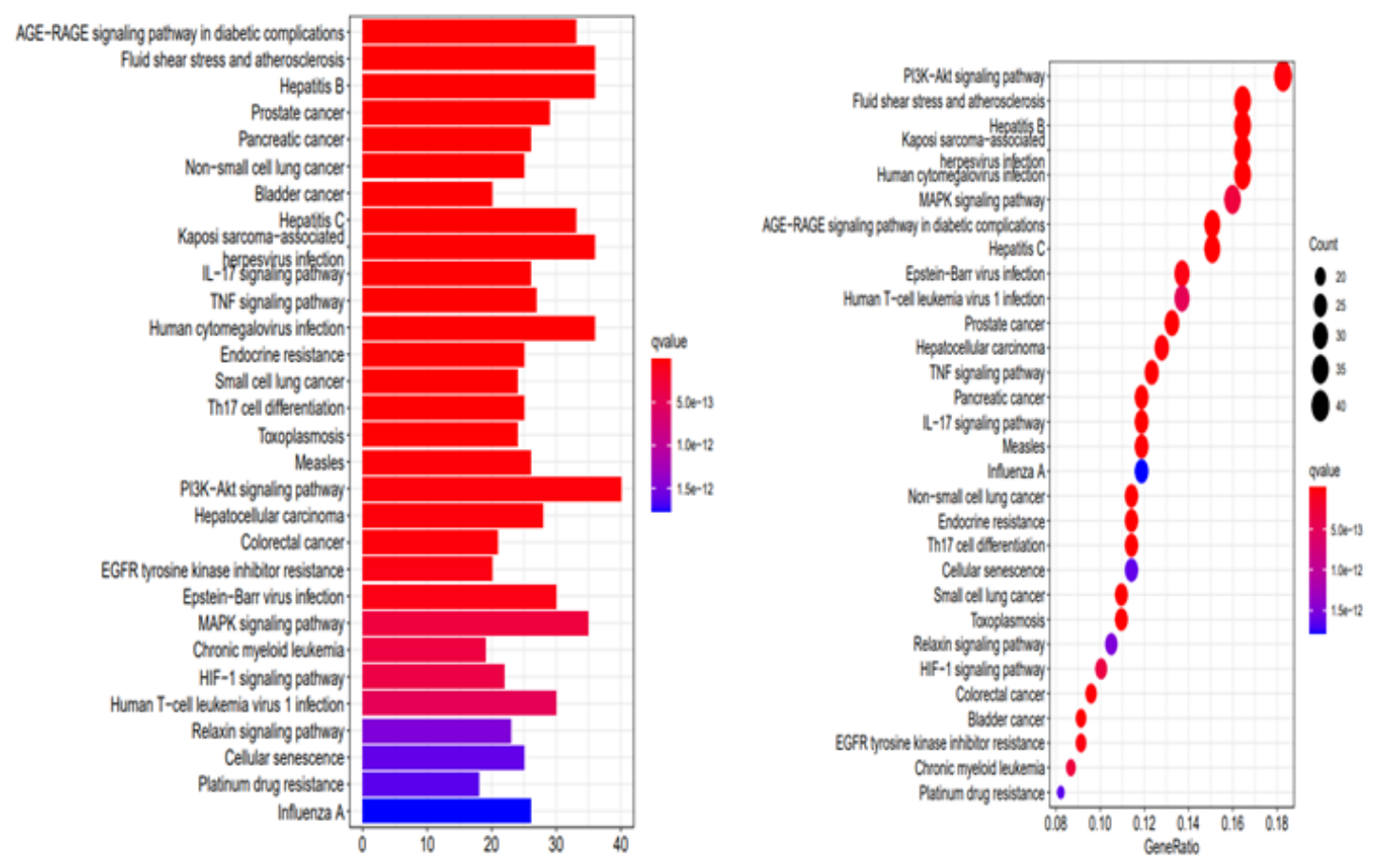

Fig. 8: KEGG enrichment analysis of the intersection target

TABLE 2: THE DOCKING RESULTS OF KEY ACTIVE INGREDIENTS AND CORE TARGET MOLECULES

\begin{tabular}{|c|c|c|c|c|}
\hline Ingredient & Target & UniProt ID & PDB ID & Affinity/(kcal/mol) \\
\hline \multirow{7}{*}{ Quercetin } & CDKN1A & P38936 & $6 \mathrm{p} 8 \mathrm{~h}$ & -7.6 \\
\hline & TP53 & P04637 & 1gzh & -6.6 \\
\hline & RB1 & P06400 & 1 gux & -8.2 \\
\hline & CCND1 & P24385 & $2 w 9 f$ & -7.1 \\
\hline & JUN & P05412 & $1 \mathrm{a} 02$ & -8.2 \\
\hline & MAPK1 & P28482 & $2 y 9 q$ & -9.0 \\
\hline & RELA & Q04206 & $3 q x y$ & -7.7 \\
\hline \multirow{2}{*}{ Kaempferol } & JUN & P05412 & $1 \mathrm{a} 02$ & -8.1 \\
\hline & RELA & Q04206 & $3 q x y$ & -7.1 \\
\hline \multirow{4}{*}{ Naringenin } & MAPK3 & P27361 & $2 z o q$ & -8.7 \\
\hline & ESR1 & P03372 & $2 b j 4$ & -8.5 \\
\hline & RELA & P27361 & $3 q x y$ & -7.2 \\
\hline & MAPK1 & P28482 & $2 y 9 q$ & -9.0 \\
\hline \multirow{3}{*}{ Formononetin } & MAPK14 & Q16539 & $3 c t q$ & -8.5 \\
\hline & ESR1 & P03372 & $2 b j 4$ & -8.6 \\
\hline & JUN & P05412 & $1 \mathrm{a} 02$ & -7.9 \\
\hline \multirow{3}{*}{ Isorhamnetin } & MAPK14 & Q16539 & $3 c t q$ & -7.6 \\
\hline & ESR1 & P03372 & $2 b j 4$ & -8.2 \\
\hline & RELA & Q04206 & $3 q x y$ & -7.1 \\
\hline \multirow{2}{*}{ Glabridin } & MAPK14 & Q16539 & $3 c t q$ & -8.7 \\
\hline & ESR1 & P03372 & 2bj4 & -7.4 \\
\hline
\end{tabular}




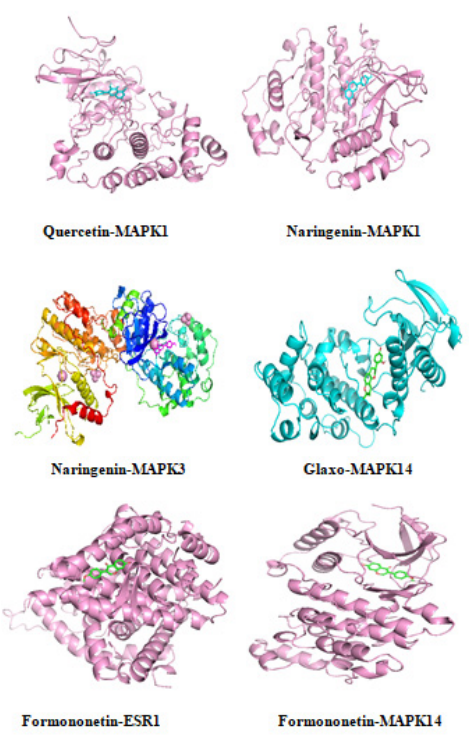

Fig. 9: Molecule docking mode diagram

In recent years, Chinese medicine has made major breakthroughs in the treatment of CHF and has unique therapeutic advantages in improving patient's clinical symptoms, improving quality of life and improving prognosis ${ }^{[7]}$. "Suwen•Ni Tiao Lun Pian" says "If the heart qi is weak and weak, it can be seen that the wheezing will continue", "Yi Lin Jiao Wong" says that "the vitality is weak, it must not reach the blood vessels and the blood vessels will stay and stasis if there is no qi.", both the importance of Qi deficiency in the occurrence and development of CHF is emphasized and the treatment of CHF should be based on supplementing temperature and yang. Modern pharmacological studies have shown that Baoyuan decoction has the effects of inhibiting the increase of ROS, inhibiting calcium ion overload, promoting cell proliferation, inhibiting cardiomyocyte apoptosis, regulating mitochondrial function and improving energy metabolism ${ }^{[8]}$.

In this study, the effective components and pharmacological mechanisms of Baoyuan decoction in the treatment of CHF were analyzed by the method of network pharmacology. By using the TCMSP database, 144 effective active ingredients of Baoyuan decoction, 253 drug targets, 9424 CHF disease related genes and 240 targets of Baoyuan decoction and CHF disease intersection were obtained. Through the analysis of the network diagram of Baoyuan decoction's active ingredients-CHF intersection target point, it can be seen that the key active ingredients of Baoyuan decoction are quercetin in licorice and astragalus; kaempferol in astragalus, licorice and ginseng; naringenin in licorice; isorhamnetin in astragalus and licorice; formononetin in licorice and astragalus; glazin in licorice; linoleic acid in cinnamon. Among them, quercetin has the effects of reducing inflammation, anti-myocardial fibrosis, anti-oxidative stress and anti-apoptosis ${ }^{[9,10]}$; kaempferol inhibits endoplasmic reticulum stress by activating the PI3K/Akt/Glycogen Synthase Kinase-3 beta (GSK-3 $\beta$ ) and Extracellular-Signal-Regulated Kinase (ERK)/MAPK pathways, down-regulates the phosphorylation of $\mathrm{p} 38$ and JUN, reduces the protein expression of Matrix Metallopeptidase 9 (MMP9) and MMP-2, and increases the level of B-cell lymphoma 2 (Bcl-2), down-regulates the levels of caspase-3 and Bcl-2-associated $\mathrm{X}$ protein (Bax) to reduce cell apoptosis, while reducing Nuclear factor erythroid 2-related factor $2(\mathrm{Nrf} 2), \mathrm{NF}-\kappa \mathrm{B}$, reduces the increase of ROS, effectively inhibiting oxidative stress, inflammation and apoptosis, thereby improving myocardial damage ${ }^{[11,12]}$; Naringenin can increase Vascular Endothelial Growth Factor (VEGF) and Basic Fibroblast Growth Factor (BFGF) protein levels, promote vascular endothelial regeneration, reduce collagen deposition and myocardial tissue damage and improve heart function. It also has anti-diabetic, antitumor and anti-inflammatory effects ${ }^{[13,14]}$; Isorhamnetin has anti-inflammatory, anti-atherosclerosis, lipidlowering, anti-hyperuricemia, anti-tumor and protective effects on cardiovascular diseases ${ }^{[15]}$; Formononetin can increase the antioxidant capacity of cardiomyocytes by reducing the content of Malondialdehyde (MDA) and up-regulating Superoxide Dismutase (SOD) activity. It can also reduce the neuronal damage induced by lipopolysaccharide and inhibit the levels of TNF- $\alpha$, IL-1 $\beta$ and IL-6 through ROD-Thioredoxin Interacting Protein (TXNIP). NLR family pyrin domain containing 3 (NLRP3) pathway inhibits the activation of NLRP3 
protein, thereby exerting anti-inflammatory and improving heart function ${ }^{[16,17]}$; Glaxo down-regulates the expression and activity of Myosin Light Chain Kinase (MLCK) through the MAPK pathway, reduces arterial intimal permeability, improves vascular endothelial function and then exerts a protective effect on the cardiovascular system ${ }^{[18]}$. It can also inhibit LowDensity Lipoprotein (LDL) oxidation and Nicotinamide Adenine Dinucleotide Phosphate (NADPH) oxidase, increase the expression of antioxidant enzymes in macrophages, stimulate DNA synthesis in endothelial cells, inhibit the proliferation of vascular smooth muscle and exert antioxidant effects to improve heart function $^{[19]}$. It shows that the material basis of Baoyuan decoction for treating CHF is mainly flavonoids such as quercetin and naringenin. It mainly improves heart function and reduces myocardial tissue damage by intervening in inflammation, oxidative stress, apoptosis and cancer pathways and protects the role of the heart.

Through the PPI network, Cytoscape 3.8.0 is used to screen out 12 core targets which includes STAT3, FOS, TP53, JUN, MAPK1, MAPK3, RELA, ESR1, RB1, CDKN1A, MAPK14, CCND1, etc. At the same time, it is closely related to Akt1, MAPK8, Vascular Endothelial Growth Factor A (VEGFA), Prostaglandin-Endoperoxide Synthase 2 (PTGS2), Peroxisome Proliferator Activated Receptor Gamma (PPARG), MYC Proto-Oncogene (MYC), Bcl-2, IL$1 \beta$, IL-4, Cyclin-Dependent Kinase (CDK) 1, CDK2, CDK4, NOS2 and other targets. As a sensor of acute and chronic stress, STAT3 plays an important role in myocardial hypertrophy, myocardial infarction and heart failure. As a transcription factor, it protects the heart by regulating mitochondria, immune function, inflammation and redox reaction ${ }^{[20]}$. MAPK1, MAPK3 and MAPK14 are important components of the MAP kinase signaling pathway, which regulate various BPs through a variety of mechanisms and interact with JNK, Bcl-2, Growth Factor Receptor Bound Protein 7 (GRB7), Akt1, IL-6, TNF, Kinase Suppressor of Ras-1 (KSR1) and other proteins in regulating inflammation, cell adhesion, proliferation and migration. T cells play an important role in regulating immunity and inhibiting cell apoptosis ${ }^{[21]}$. NF- $\kappa \beta$ is a nuclear protein factor that regulates gene expression and plays an important role in the regulation of gene transcription such as cell proliferation, differentiation, inflammation and immune response. As a member of the NF- $\kappa \beta$ family, RELA plays a central role in innate immunity ${ }^{[22]}$.

GO enrichment analysis results show that Baoyuan decoction mainly regulates nutritional response, oxidative stress response, lipopolysaccharide response, steroid hormone response, ROS in inflammation, apoptosis, signal pathways and other BPs, exert MF such as DNA binding transcription factors, amides, RNA polymerase II specific DNA binding transcription factors, ubiquitin-like protein ligases, growth factors and other MFs to treat CHF. The role of KEGGenriched molecular results showed that Baoyuan decoction is mainly related to PI3K-Akt, MAPK, AGERAGE, cancers, TNF, IL-17, NF- $\mathrm{B}$ and apoptosis signaling pathways. The PI3K-Akt signaling pathway plays an important role in regulating the growth and proliferation of cardiomyocytes, inhibiting apoptosis, inhibiting inflammatory factors and regulating energy metabolism ${ }^{[23]}$. The MAPK signaling pathway is a key pathway that regulates various BPs. It is composed of the ERK, p38 and JNK signaling pathways. It is generally believed that the activation of ERK can promote cell proliferation and growth, while the activation of p38 and JNK is generally considered to promote cell apoptosis ${ }^{[24]}$. p38 MAPKs are the key signal molecules that cells respond to external stimuli and regulate pro-inflammatory cytokines. Activated p38 can reduce cell activity and induce oxidative stress to cause heart failure and other cardiovascular diseases $^{[25]}$. The AGE-RAGE signaling pathway can regulate the pathogenesis of cardiovascular diseases by regulating the effects of atherosclerosis, mitochondrial dysfunction, oxidative stress, calcium homeostasis and cytoskeletal function ${ }^{[26]}$. Increased levels of AGEs can further enhance tissue damage and inflammation. RAGE receptors can interact with a variety of ligands to activate NF- $\kappa \beta$, the main regulator of inflammation and stimulate the phosphorylation of RELA induced by Transforming Growth Factor beta (TGF- $\beta$ ) and TNF- $\alpha$. The expression of collagen type I further affects vascular remodeling ${ }^{[27]}$. Sympathetic nerve excitation and excessive activation of the Renin-AngiotensinAldosterone System (RAAS) system can induce the release of inflammatory factors. TNF- $\alpha$, IL-1 $\beta$, IL-6, C-Reactive Protein (CRP) and other pro-inflammatory factors promote the release of ROS and Reactive Nitrogen Species (RNS), reduce sarcoplasmic reticulum calcium load and reduce Adenosine Triphosphate (ATP) production, affect myocardial metabolism, aggravate ventricular hypertrophy and myocardial fibrosis, promote myocardial cell apoptosis and accelerate the development of $\mathrm{CHF}^{[28,29]}$.

The relationship between cancer and CHF has attracted more and more attention. The results of 
certain tumor markers such as cancer antigen 125 (CA125), Cytokeratin 19 Fragment Antigen (CYFRA 21-1), carcinoembryonic antigen (CEA) and Cancer Antigen 19-9 (CA19-9) are positively correlated with the severity of $\mathrm{CHF}^{[30]}$. Long-term radiotherapy and anthracycline chemotherapy for cancer patients increase the incidence of cardiovascular disease, which is quantitatively dependent ${ }^{[31]}$. Genetics, inflammation and microcirculation changes are all important risk factors for heart failure and cancer. Inflammation and oxidative stress are important pathways for CHF and cancer. When oxidative stress and inflammation are activated, inflammatory factors TNF- $\alpha$ and IL- 6 can aggravate myocardial tissue damage, endothelial cells produce ROS, reduce Nitric Oxide (NO) levels, induce actin phosphorylation and cause damage to myocardial diastolic function. Inflammation can also regulate the cell cycle and Epithelial-Mesenchymal Transition (EMT) of Cancer Stem Cells (CSCs). The activated NF- $\kappa \beta$ and STAT3 pathways promote the proliferation of CSCs. TNF- $\alpha$, IL- 6 and TGF- $\beta$ induce tumor cell migration and activation of cancer cells ${ }^{[32]}$, suggesting an interaction between heart failure and cancer.

In summary, this study revealed the main material basis and mechanism of action of Baoyuan decoction by analyzing network pharmacology results. It was discovered that Baoyuan decoction material basis is flavonoids, saponins and alcohol compounds, which mainly act on core targets such as MAPK1, MAPK3, MAPK14, ESR1, etc., and play through PI3K-Akt, MAPK, AGE-RAGE, TNF, cancer and other pathways, inhibit inflammation, anti-oxidation, inhibit cell apoptosis, regulate immune response, anti-tumor and other functions, so as to work for CHF cure. At the same time, it was found that the target of Baoyuan decoction is closely related to a variety of cancer pathways, suggesting that Baoyuan decoction may reduce the death of non-cardiovascular diseases caused by cancer by intervening in cancer pathways, thereby reducing the mortality of CHF.

\section{Funding:}

This work was supported by National Natural Science Foundation of China (81774224).

\section{Conflicts of interest:}

The authors declared no conflict of interest.

\section{REFERENCES}

1. Anhua W, Juan L. Pharmacologic therapy updates of 2018 guidelines for the diagnosis and treatment of heart failure in China. Her Med 2019;38(05):539-43.
2. Krysztofiak H, Wleklik M, Migaj J, Dudek M, Uchmanowicz I, Lisiak M, et al. Cardiac cachexia: a well-known but challenging complication of heart failure. Clin Interv Aging 2020;15:2041.

3. The writing committee of the report on cardiovascular health and diseases in China. Report on cardiovascular health and disease in China 2019: An updated summary. Chin Circ J 2020;35(09):833-54.

4. $\mathrm{Ma} \mathrm{L}, \mathrm{Wu} \mathrm{Y}, \mathrm{Chen} \mathrm{W}$. Highlights of China cardiovascular disease report 2018. Chin J Hypertens 2019;27(8):712-6.

5. Xu H, Qi XJ, Fang CS. Study on the mechanism of Zhenwu decoction in treating chronic heart failure based on network pharmacology. Chin J New Drugs Clin Rem 2020;37(15):180111.

6. Lu AN, Wang DL, Zhao F, Chen RJ, Chen WQ, Zheng HB, et al. Mechanism of Shaoyao decoction in treatment of ulcerative colitis based on network pharmacology and molecular docking technology. Chin Tradit Herb Drugs 2020;51(23):6035-44.

7. Guidelines for rational use of drugs of heart failure ( $2^{\text {nd }}$ Edition). Chin J Front Med Sci 2019;11(7):1-78.

8. Shu ZL, Zeng KW, Ma XL, Tu PF, Li J, Jiang Y. Active compounds from Baoyuan decoction with myocardial protection activities and their potential targets. China J Chin Mater Med 2016;41(5):922-7.

9. Andres S, Pevny S, Ziegenhagen R, Bakhiya N, Schäfer B, Hirsch-Ernst KI, et al. Safety aspects of the use of quercetin as a dietary supplement. Mol Nutr Food Res 2018;62(1):1700447.

10. Jiao M, Zhong HY, Chen KY, Wu TF. Quercetin improved myocardial fibrosis in rats with chronic heart failure via TGF $\beta 1 /$ Smad3 signaling pathways. Prog Anat Sci 2020;26(4):391-5.

11. Abdullah A, Ravanan P. Kaempferol mitigates endoplasmic reticulum stress induced cell death by targeting caspase $3 / 7$. Sci Rep 2018;8(1):1-5.

12. Zhang L, Guo Z, Wang Y, Geng J, Han S. The protective effect of kaempferol on heart via the regulation of Nrf2, NF- $\kappa \beta$, and PI3K/Akt/GSK-3 $\beta$ signaling pathways in isoproterenolinduced heart failure in diabetic rats. Drug Dev Res 2019;80(3):294-309.

13. Xin W, Lei L, Yuanyou C, Xiao W. Study on the promoting effect and mechanism of naringenin on angiogenesis in rats with myocardial infarction. J New Med 2020;51(12):915-21.

14. Choi J, Lee DH, Jang H, Park SY, Seol JW. Naringenin exerts anticancer effects by inducing tumor cell death and inhibiting angiogenesis in malignant melanoma. Int J Med Sci 2020;17(18):3049-57.

15. Gong G, Guan YY, Zhang ZL, Rahman K, Wang SJ, Zhou S, et al. Isorhamnetin: a review of pharmacological effects. Biomed Pharmacother 2020;128:110301.

16. Tay KC, Tan LT, Chan CK, Hong SL, Chan KG, Yap WH, et al. Formononetin: a review of its anticancer potentials and mechanisms. Front Pharmacol 2019;10:820.

17. Yu X, Su CP, Wang X, Fan A, Wang S. Protective effect of kaempferol and formononetin against myocardial oxidative stress damage of $\mathrm{H} 9 \mathrm{c} 2$ cells. Liaoning J Tradit Chin Med 2020;47(3):154-6.

18. Deutch MR, Grimm D, Wehland M, Infanger M, Krüger M. Bioactive candy: Effects of licorice on the cardiovascular system. Foods 2019;8(10):495.

19. Wang G, Sun G, Wang Y, Yu P, Wang X, Zhou B, et al. Glabridin attenuates endothelial dysfunction and permeability, possibly via the MLCK/p-MLC signaling pathway. Exp Ther Med 2019;17(1):107-14. 
20. Kurdi M, Zgheib C, Booz GW. Recent developments on the crosstalk between STAT3 and inflammation in heart function and disease. Front Immunol 2018;9:3029.

21. Yue J, Lopez JM. Understanding MAPK signaling pathways in apoptosis. Int J Mol Sci 2020;21(7):2346.

22. Yang L, Deng J, Deng ZH. Research progress on the relationship between NFkB and its gene polymorphism and inflammation and tumor. Guizhou Med J 2016;40(10):1098-100.

23. Hao Q, Zhang F, Wang Y, Li Y, Qi X. Cardiac contractility modulation attenuates chronic heart failure in a rabbit model via the PI3K/AKT pathway. Biomed Res Int 2020.

24. Lu M, Wang Y, Zhan X. The MAPK pathway-based drug therapeutic targets in pituitary adenomas. Front Endocrinol 2019;10:330.

25. Zhang S, Wu Y, Li X. Protective Effects of p38 MAPK inhibitor combined with coenzyme Q10 on myocardial cells under oxidative stress. J Zhengzhou Univ 2019;54(5):699-703.

26. Lee TW, Kao YH, Chen YJ, Chao TF, Lee TI. Therapeutic potential of vitamin D in AGE/RAGE-related cardiovascular diseases. Cell Mol Life Sci 2019;76(20):4103-15.

27. Peng Y, Kim JM, Park HS, Yang A, Islam C, Lakatta EG, et al. AGE-RAGE signal generates a specific NF- $\kappa$ B RelA "barcode" that directs collagen I expression. Sci Rep 2016;6(1):1-10.

28. Quan ZH, Liu L, Ai M, Jin J. Research progress of Chinese medicine inhibiting inflammatory markers in intervention of chronic heart failure. Int J Lab Med 2016;37(8):1092-94.
29. Fan H, Hu ZG. Research Progress on the inflammatory mechanism of chronic heart failure. Chin J Clin Ration Drug Use 2019;12(10):180-1.

30. Shi C, van der Wal HH, Sillje HH, Dokter MM, van den Berg $\mathrm{F}$, Huizinga L, et al. Tumour biomarkers: association with heart failure outcomes. J Intern Med 2020;288(2):207-18.

31. Boekel NB, Schaapveld M, Gietema JA, Russell NS, Poortmans P, Theuws JC, et al. Cardiovascular disease risk in a large, population-based cohort of breast cancer survivors. Int J Radiat Oncol Biol Phys 2016;94(5):1061-72.

32. Ausoni S, Azzarello G. Development of cancer in patients with heart failure: how systemic inflammation can lay the groundwork. Front Cardiovasc Med 2020;7:1-10.

This is an open access article distributed under the terms of the Creative Commons Attribution-NonCommercial-ShareAlike 3.0 License, which allows others to remix, tweak, and build upon the work non-commercially, as long as the author is credited and the new creations are licensed under the identical terms

This article was originally published in a special issue, "Novel Therapeutic Approaches in Biomedicine and Pharmaceutical Sciences" Indian J Pharm Sci 2021:83(6) Spl Issue "19-28" 